\title{
Crystal structure of cis-diammine-bis(nitrito)-platinum(II)
}

Volker Kahlenberg, ${ }^{\mathrm{a} *}$ Thomas Gelbrich, $^{\mathrm{b}}$ Richard Tessadri $^{\mathrm{a}}$ and Frederik Klauser $^{\mathrm{c}}$

${ }^{a}$ University of Innsbruck, Institute of Mineralogy \& Petrography, Innrain 52, A-6020 Innsbruck, Austria, ${ }^{\mathbf{b}}$ University of Innsbruck, Institute of Pharmacy, Innrain 80, 6020 Innsbruck, Austria, and ${ }^{c}$ MED-EL Medical Electronics, Fürstenweg 77a, A-6020 Innsbruck, Austria

Correspondence email: volker.kahlenberg@uibk.ac.at 


\section{Supporting information}

\section{S1. Hydrogen-bonded structures}

Table S1 Geometry for intermolecular hydrogen bonds $\left(\AA,^{\circ}\right)$ used for the definition of the HBS (Figure 5b) of trans- $\mathrm{Pt}\left(\mathrm{NH}_{3}\right)_{2}\left(\mathrm{NO}_{2}\right)_{2}$ (Madarász et al., 2007).

\begin{tabular}{lcccc}
\hline$D-\mathrm{H} \cdots A$ & $D-\mathrm{H}$ & $\mathrm{H} \cdots A$ & $D \cdots A$ & $\angle(D-\mathrm{H} \cdots A)$ \\
\hline $\mathrm{N} 1-\mathrm{H} 1 \cdots \mathrm{O} 2^{\mathrm{i}}$ & 0.90 & 2.34 & $3.1479(7)$ & 150 \\
$\mathrm{~N} 1-\mathrm{H} 2 \cdots \mathrm{O} 1^{\mathrm{ii}}$ & 0.89 & 2.25 & $3.0443(7)$ & 147 \\
$\mathrm{~N} 1-\mathrm{H} 3 \cdots \mathrm{O} 1^{\mathrm{iii}}$ & 0.88 & 2.49 & $3.1870(7)$ & 136 \\
\hline Symmetry codes: (i) $1 / 2-\mathrm{x},-1 / 2+\mathrm{y}, 1-\mathrm{z} ;$ (ii) $1 / 2-\mathrm{x}, 1 / 2+\mathrm{y}, 1-\mathrm{z} ;$ (iii) $1 / 2-\mathrm{x}, 1 / 2+\mathrm{y},-\mathrm{z}$.
\end{tabular}

Table S2 Geometry for intermolecular hydrogen bonds $\left(\AA,^{\circ}\right)$ used for the definition of the HBS (Figure 5b) of trans-Pd( $\left.\mathrm{NH}_{3}\right)_{2}\left(\mathrm{NO}_{2}\right)_{2}$ (Madarász et al., 2007).

\begin{tabular}{lllll}
\hline$D-\mathrm{H} \cdots A$ & $D-\mathrm{H}$ & $\mathrm{H} \cdots A$ & $D \cdots A$ & $\angle(D-\mathrm{H} \cdots A)$ \\
\hline $\mathrm{N} 1-\mathrm{H} 1 \cdots \mathrm{O} 2^{\mathrm{i}}$ & 0.89 & 2.15 & $3.0339(6)$ & 170 \\
$\mathrm{~N} 1-\mathrm{H} 2 \cdots \mathrm{O} 2^{\mathrm{ii}}$ & 0.89 & 2.21 & $3.0846(6)$ & 168 \\
$\mathrm{~N} 1-\mathrm{H} 3 \cdots \mathrm{O} 1^{\mathrm{iii}}$ & 0.89 & 2.26 & $3.1361(6)$ & 169 \\
\hline
\end{tabular}

Symmetry codes: (i) x, 1+y, $z$; (ii); 1+x, 1+y, z (iii) 1-x, $-y, 1-z$. 


\section{S2. Additional diagrams}

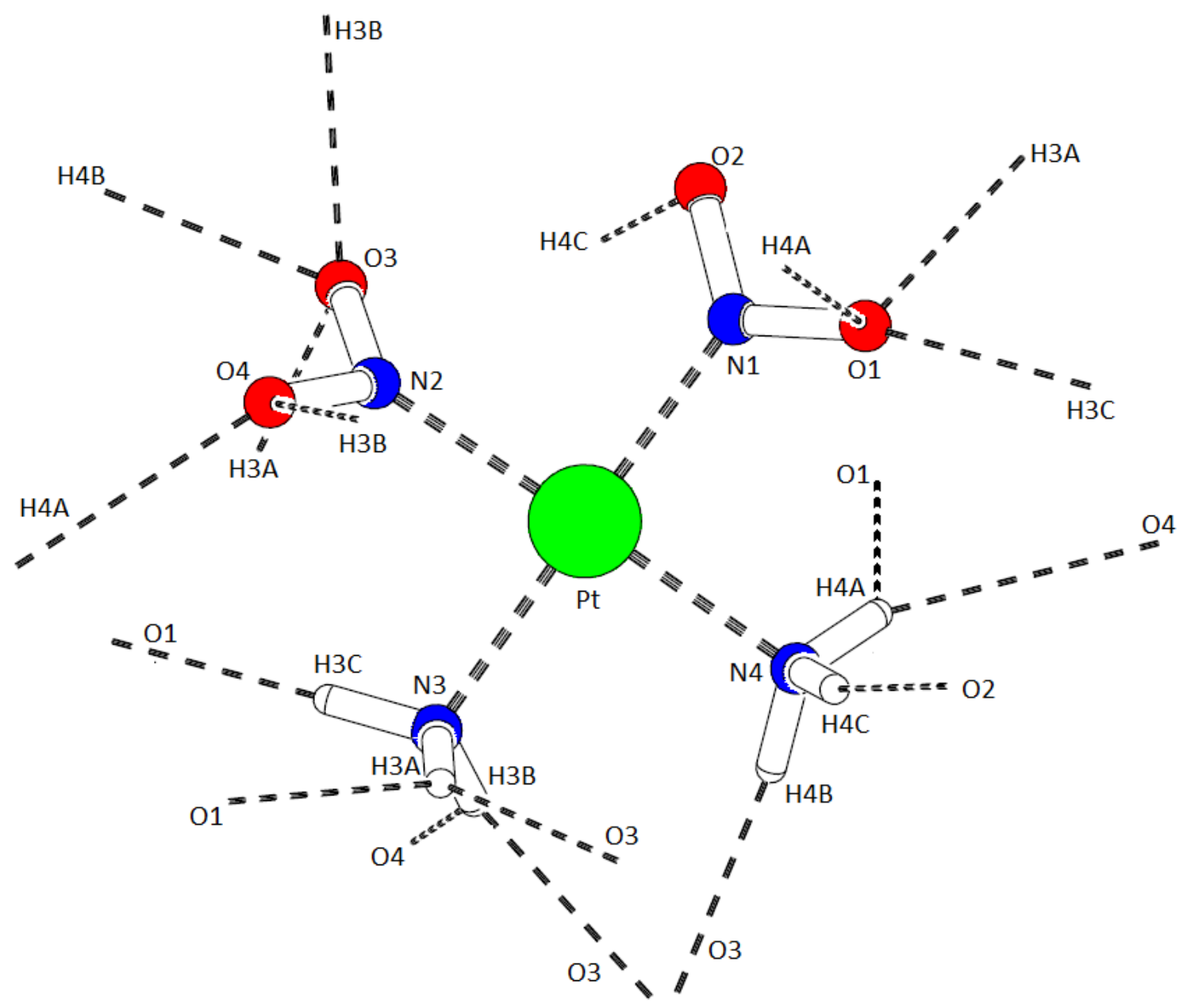

Figure S1 Intermolecular $\mathrm{N} 1-\mathrm{H} 1 \cdots \mathrm{O} 2$ interactions in cis- $\mathrm{Pt}\left(\mathrm{NH}_{3}\right)_{2}\left(\mathrm{NO}_{2}\right)_{2}$. 


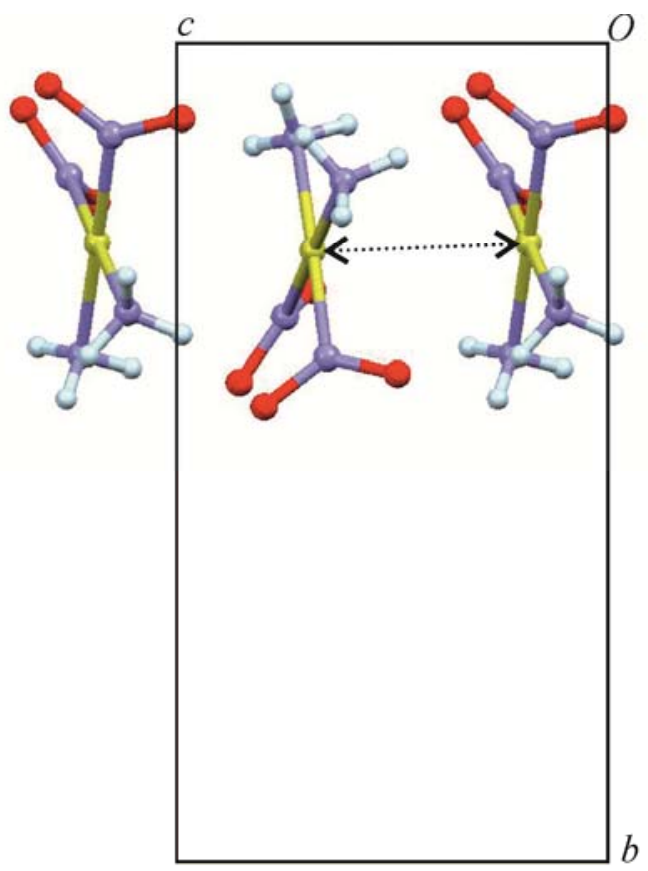

a)

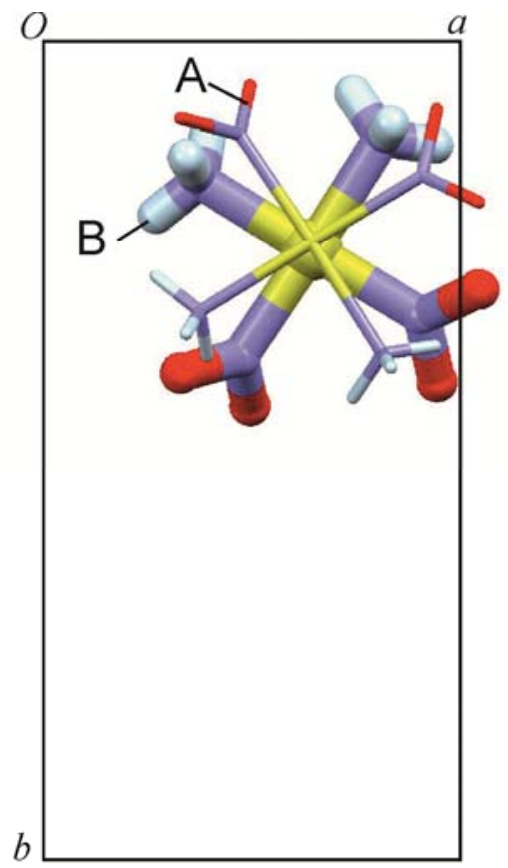

b)

Figure S2 Stack of cis- $\mathrm{Pt}\left(\mathrm{NH}_{3}\right)_{2}\left(\mathrm{NO}_{2}\right)_{2}$ molecules propagating parallel to the $c$ axis: (a) view of a single stack along [100] - the double arrow indicates the shortest Pt $\cdots$ Pt distance (3.549 Å); (b) view of a single stack along [001], showing two neighbouring molecules (denoted A and B) which are related to one another by a glide mirror operation. The two molecules have been drawn in different styles for enhanced clarity.

\section{References}

Madarász, J., Bombicz, P., Mátyás, C., Réti, F., Kiss, G. \& Pokol, G. (2007). Thermochim. Acta 490, 51-59. 